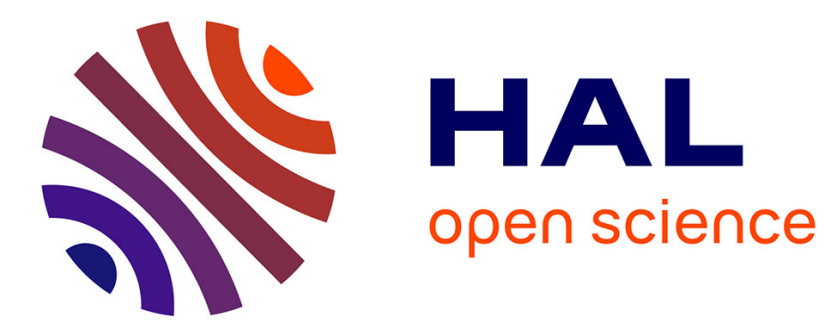

\title{
Terahertz Intersubband Electroluminescence from Nonpolar m-Plane ZnO Quantum Cascade Structures
}

Bo Meng, Borislav Hinkov, Nolwenn Marie L. Biavan, Hanh Hoang, Denis

Lefebvre, Maxime Hugues, David Stark, Martin Franckié, Almudena Torres-Pardo, Julen Tamayo-Arriola, et al.

\section{To cite this version:}

Bo Meng, Borislav Hinkov, Nolwenn Marie L. Biavan, Hanh Hoang, Denis Lefebvre, et al.. Terahertz Intersubband Electroluminescence from Nonpolar m-Plane ZnO Quantum Cascade Structures. ACS photonics, 2021, 8 (1), pp.343-349. 10.1021/acsphotonics.0c01641 . hal-03423464

\author{
HAL Id: hal-03423464 \\ https://hal.science/hal-03423464
}

Submitted on 22 Nov 2021

HAL is a multi-disciplinary open access archive for the deposit and dissemination of scientific research documents, whether they are published or not. The documents may come from teaching and research institutions in France or abroad, or from public or private research centers.
L'archive ouverte pluridisciplinaire HAL, est destinée au dépôt et à la diffusion de documents scientifiques de niveau recherche, publiés ou non, émanant des établissements d'enseignement et de recherche français ou étrangers, des laboratoires publics ou privés. 


\section{Terahertz Intersubband Electroluminescence from Nonpolar m-Plane ZnO Quantum Cascade Structures}

Bo Meng,* Borislav Hinkov,* Nolwenn Marie L. Biavan, Hanh T. Hoang, Denis Lefebvre, Maxime Hugues, David Stark, Martin Franckié, Almudena Torres-Pardo, Julen Tamayo-Arriola, Miguel M. Bajo, Adrian Hierro, Gottfried Strasser, Jérôme Faist,* and Jean M. Chauveau*

Cite This: ACS Photonics 2021, 8, 343-349

Read Online

\section{ACCESS \\ Wl Metrics \& More \\ Article Recommendations \\ Supporting Information}

ABSTRACT: The $\mathrm{ZnO}$-based heterostructures are predicted to be promising candidates for optoelectronic devices in the infrared and terahertz $(\mathrm{THz})$ spectral domains owing to their intrinsic material properties. Specifically, the large $\mathrm{ZnO}$ LOphonon energy reduces the thermally activated LO-phonon scattering, which is predicted to greatly improve the temperature performance of $\mathrm{THz}$ quantum cascade lasers. However, to date, no experimental observation of intersubband emission from $\mathrm{ZnO}$ optoelectronic devices has been reported. Here, we report the observation of $\mathrm{THz}$ intersubband electroluminescence from $\mathrm{ZnO} / \mathrm{Mg}_{x} \mathrm{Zn}_{1-x} \mathrm{O}$ quantum cascade structures grown on a nonpolar m-plane $\mathrm{ZnO}$ substrate up to room temperature. The electroluminescence peak shows a line width of $\sim 20 \mathrm{meV}$ at a center frequency of $\sim 8.5 \mathrm{THz}$ at $110 \mathrm{~K}$, which is not accessible for GaAs-based quantum cascade structures because of the reststrahlen band absorption from 8 to $9 \mathrm{THz}$. This result is an important step toward the realization of $\mathrm{ZnO}$-based $\mathrm{THz}$ quantum cascade lasers.

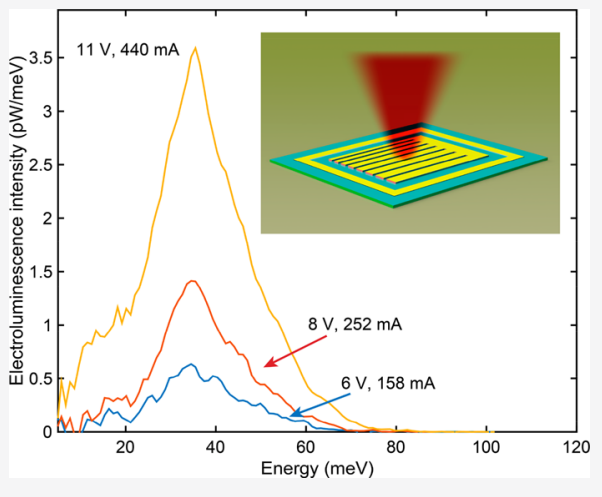

KEYWORDS: $\mathrm{THz}$, quantum cascade laser, nonpolar $\mathrm{ZnO}$, electroluminescence, molecular beam epitaxy

$\mathrm{T}$ he terahertz $(\mathrm{THz})$ frequency spectrum, defined as the frequency range from 0.1 to $10 \mathrm{THz}$, has attracted great attention among many research groups owing to its potential for applications including, but not limited to, noninvasive characterization, defect monitoring, security screening, astronomy, explosive material detection, and time domain spectroscopy. ${ }^{1-3}$ Highly required for these applications are compact $\mathrm{THz}$ sources, with milliwatt-level output power, continuous wave operation, and broad tunability. Many electronic and photonic approaches have been proposed and investigated for THz sources so far, such as Schottky diode multipliers, ${ }^{4}$ Gunn oscillators, ${ }^{5}$ resonant-tunneling diodes (RTDs), ${ }^{6}$ as well as photonic devices based on nonlinear or photoconductive effects. ${ }^{7}$ Though useful in their specific fields, their application is still limited by their intrinsic properties and structures. Among the $\mathrm{THz}$ sources, the quantum cascade laser (QCL), a compact injection laser based on semiconductor heterostructures, has become the most appealing choice for realworld applications thanks to its compactness, high power output, and flexible design capabilities. In recent years, intensive efforts have been devoted to enhance the performance of $\mathrm{THz}$ QCLs. Nowadays, THz QCLs have already shown high output powers (several hundred $\mathrm{mW}$ both in pulsed and continuous wave (CW) operations) and lasing emission ranging from 1 to $6 \mathrm{THz}$. Recently, thermoelectrically cooled $\mathrm{THz}$ QCLs operating up to $210 \mathrm{~K}$ have been demonstrated thanks to a precise design optimized using nonequilibrium Green's function simulations. ${ }^{8,9}$ Nevertheless, despite the significant progress, $\mathrm{THz}$ QCL operation at roomtemperature (RT) is still lacking. Besides structure optimization using the conventional GaAs/AlGaAs material system, other potential material systems have also been proposed and investigated, such as $\mathrm{Si} / \mathrm{SiGe}{ }^{10} \mathrm{GaN} / \mathrm{AlGaN},{ }^{11}$ and more recently $\mathrm{ZnO} / \mathrm{ZnMgO} .^{12-14}$ Compared with the classical GaAs-based approach, nonpolar material systems such as $\mathrm{Si}$ / $\mathrm{SiGe}$ are attractive because of the weaker electron-phonon interaction, enabling robust population inversion with increasing operating temperature. ${ }^{15}$ As an alternative strategy, the wide-bandgap $\mathrm{ZnO}$ or $\mathrm{GaN}$ material systems have recently been proposed, offering several advantages including large LO phonon energy and higher conduction band offset (CBO). This large LO-phonon energy will strongly reduce the thermally activated nonradiative LO-phonon scattering process, postulated to be the main scattering mechanism at high temperature, thus, significantly improving the predicted temperature performance of THz QCLs. ${ }^{16}$ Meanwhile, though

Received: October 23, 2020

Published: December 30, 2020 
GaAs-based THz QCLs have been quite successful in the low frequency range $(1-5 \mathrm{THz})$, further extending the spectral range to higher frequencies is limited by the intrinsic reststrahlen band of the material (around 8 to $9 \mathrm{THz}$ for $\mathrm{GaAs}$, in relation with the $36 \mathrm{meV} \mathrm{LO}$ phonon energy). This issue is naturally overcome by using material systems with large phonon energies. For instance, the $50 \mathrm{meV}$ TO phonon energy in $\mathrm{ZnO}$ leads to a reststrahlen band above $12 \mathrm{THz}$. The development of commercially available $\mathrm{ZnO}$ substrates and/or the excellent $\mathrm{ZnO} / \mathrm{ZnMgO}$ heterostructure growth control have enabled the realization of UV LEDs, ${ }^{17}$ high mobility twodimensional electron gases ${ }^{18}$ and integrated devices. ${ }^{19}$ More recently, the intersubband absorption has been observed on nonpolar $\mathrm{ZnO}$ substrates, ${ }^{20}$ and more complex physical phenomena have also been investigated. ${ }^{21,22}$ This work represents a new step toward the realization of $\mathrm{ZnO}$-based $\mathrm{THz}$ QCLs. Indeed, we report for the first time $\mathrm{THz}$ intersubband electroluminescence from $\mathrm{ZnO} / \mathrm{Zn}_{1-x} \mathrm{Mg}_{x} \mathrm{O}$ quantum cascade structures based on a four-well scheme.

\section{RESULTS}

Figure 1 displays the conduction band profile (under an applied electric field of $130 \mathrm{mV} /$ period) with the relevant

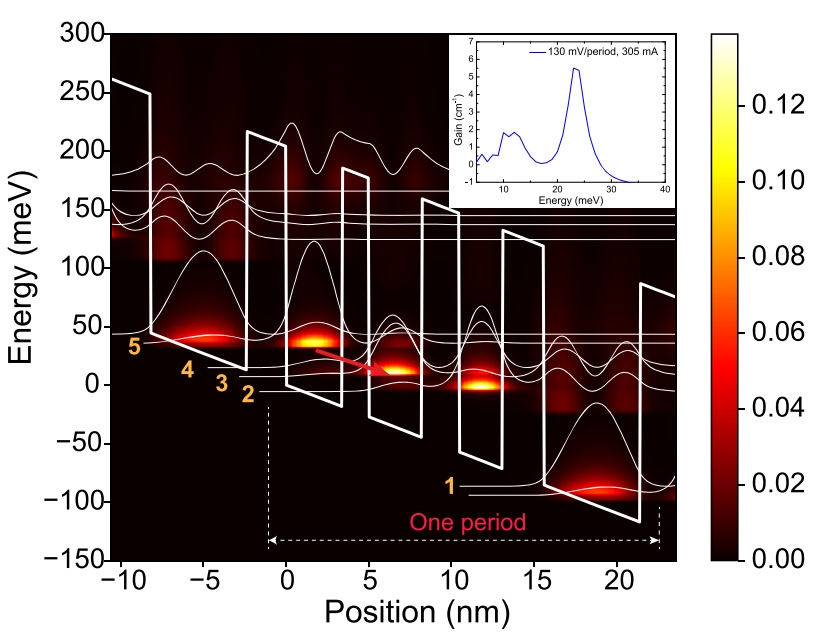

Figure 1. Density of state and Wannier-Stark wave functions shifted by their eigen energies of the structure ZOE1 under an applied electric field of $130 \mathrm{mV} /$ period at a lattice temperature of $110 \mathrm{~K}$. Here, the wave functions are only shown for clarity and are not used in the actual simulations. ${ }^{23}$ The layer sequence of one period of the structure, in nanometers, left to right and starting from the injection barrier is $2.5 / 3.6 / 1.7 / 3.4 / 2.4 / 2.75 / 2.7 / \underline{6.15}$ where the $\mathrm{Zn}_{88} \mathrm{Mg}_{12} \mathrm{O}$ barriers are in bold, while normal characters refer to $\mathrm{ZnO}$. The center of the underlined layer was doped with Ga to $n_{\mathrm{d}}=3 \times 10^{18} \mathrm{~cm}^{-3}$. The radiative transition is labeled with a red arrow. The inset shows the corresponding gain spectrum calculated by the nonequilibrium Green's function.

Wannier-Stark states at $110 \mathrm{~K}$ of the studied quantum cascade structure. The structure has been simulated using a fully selfconsistent nonequilibirum Green's function (NEGF) model, taking into account the Hartree potential calculated by the Poisson equation. ${ }^{23}$ The design is based on four quantum wells (QWs) per period, where the narrowest well spatially separates the two active wells from the phonon extractor well. ${ }^{24,25}$ The inset shows the corresponding simulated gain spectrum with an average interface roughness fluctuation height of $0.1 \mathrm{~nm}$ and a correlation length of $10 \mathrm{~nm}$. The design features a gain peak at $\sim 24 \mathrm{meV}$, corresponding to the transition between levels 5 and 4 in the Wannier-Stark basis. The lower laser level 4 is rapidly depopulated by the strong elastic scattering into levels 3 and 2, ensuring a short lower laser state lifetime. The fast carrier extraction from the level 2 by the LO-phonon scattering in the extraction well effectively suppresses the thermal backfilling to level 4, important for high temperature operation. Meanwhile, the reduced coupling between the level 5 and the extraction well due to their spatial separation significantly suppresses parasitic currents and enables a larger current dynamic range. Moreover, the broader width of the miniband formed by levels $2-4$ as well as the very fast phonon emission rate relaxes the energy alignment with the LO-phonon extraction well. As a result, the designed structure exhibits more robustness to thickness fluctuations. ${ }^{26}$ Note that because of the strength of the Fröhlich interaction, corrections to the position of the energy states due to the scattering are large and makes the NEGF simulation much better adapted to this material system than approaches based on rate equations where the self-energy corrections are neglected.

Starting from the $\mathrm{ZnO}$ substrate, the sample structure consists of a $500 \mathrm{~nm} \mathrm{ZnO}: \mathrm{Ga}$ bottom waveguide before the active region. The active region containing 100 periods of the 4 QWs design is detailed in Figure 1. The structure ends by a $150 \mathrm{~nm} \mathrm{ZnO:Ga} \mathrm{top} \mathrm{waveguide} \mathrm{layer.} \mathrm{To} \mathrm{avoid} \mathrm{any} \mathrm{Ga}$ diffusion from the waveguides, a $20 \mathrm{~nm}$ thick nonintentionally doped (nid) $\mathrm{ZnO}$ layer was inserted on both sides of the active region. In accordance with the design, the $\mathrm{Mg}$ content and the active region doping were carefully fixed to $12 \%$ and $3 \times 10^{18}$ $\mathrm{cm}^{-3}$, respectively. The reason for such a high doping level is due to the large ionization energy of the Ga dopant.

Prior to sample processing, structural characterizations have been carried out to assess the active region quality, periodicity and thicknesses of the constituting layers. Figure 2a displays the X-ray diffraction (XRD) spectrum for the (100) reflection. The highest intensity peak located at $2 \theta=31.77^{\circ}$ corresponds to the (100) reflection of the $\mathrm{ZnO}$ substrate. On both sides of this main peak, we can observe intense and regularly spaced peaks. They are superlattice peaks related to the repetition of the active region period. The spacing between the superlattice peaks gives access to the active region period thickness, which is found to be $23.8 \mathrm{~nm}$. The small difference compared to the nominal value $(25.2 \mathrm{~nm})$ ensures that each single layer within the active region has less than $1 \mathrm{ML}$ deviation from the nominal value. In addition, the sharpness of the superlattice peaks reveals a good period reproducibility across the entire structure. $^{27}$ Scanning transmission electron microscopy (STEM) was performed to evaluate the stack reproducibility and the interface quality at the atomic scale. In order to reveal the chemical contrasts, a high angle annular dark field detector (HAADF) was performed. Figure $2 b$ represents a large view of the first 15 periods of the active region on top of the bottom $\mathrm{ZnO}: \mathrm{Ga}$ waveguide. At this low magnification, we can observe the regularity of the stack with perfectly flat interfaces and constant layer thicknesses over several hundreds of nanometers along the growth direction but also along the layers plane. The inset displays a high-resolution image of a single active region period. The $\mathrm{ZnO}$ and $\mathrm{Zn}_{88} \mathrm{Mg}_{12} \mathrm{O}$ layers can be easily distinguished thanks to the chemical contrast (in light and dark contrast, respectively), while the regular point grid corresponds to the atomic columns. The interfaces are flat along the [0001] direction and abrupt at the atomic scale 

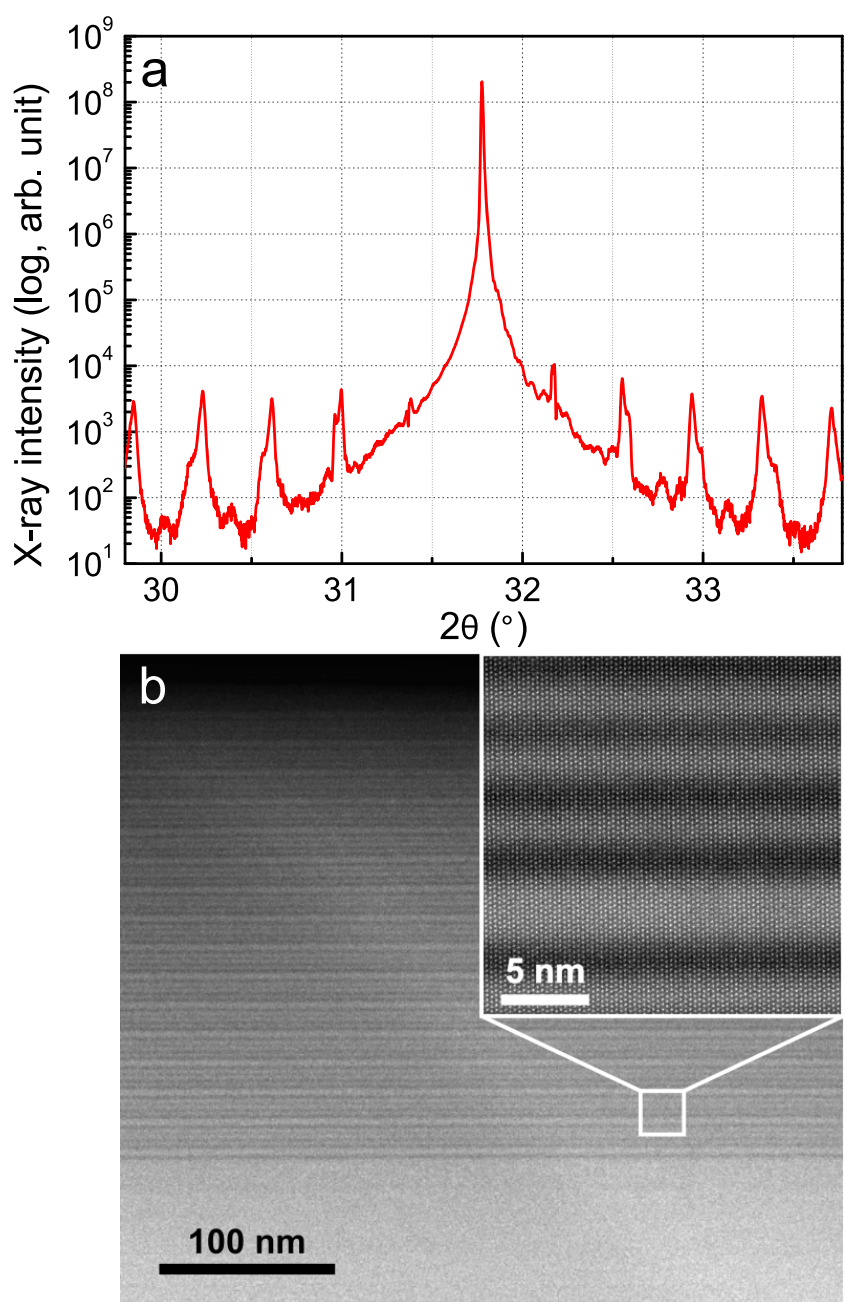

Figure 2. (a) High-resolution X-ray diffraction spectrum measured around the (100) $\mathrm{ZnO}$ reflection. (b) Scanning transmission electron microscopy image with high angle annular dark field detector in the cross-section of $\mathrm{ZnO} / \mathrm{Zn}_{88} \mathrm{Mg}_{12} \mathrm{O}$ cascade structure along the [1120] zone axis. The $\mathrm{ZnO}$ and $\mathrm{Zn}_{88} \mathrm{Mg}_{12} \mathrm{O}$ appear in light and dark contrast, respectively. Inset: zoom-in view of one active region period.

without any sign of $\mathrm{Mg}$ segregation or out-diffusion. The same thicknesses were found for the first and the last periods, which proves the high growth stability. This is in line with the sharp superlattice peaks observed on the XRD spectrum. This represents an important achievement since it demonstrates that molecular beam epitaxy of $\mathrm{ZnO}$ can match the very high requirements necessary for quantum cascades growth.

Since the intersubband nature of the emission only allows the generation of TM-polarized light, ${ }^{28}$ grating structures are needed for light outcoupling. ${ }^{29}$ Previous work showed that for $\mathrm{THz}$ emitters, the light outcoupling can be more flexible than strictly following the Bragg condition. Devices were thus fabricated using the square mesa configuration and topside grating into the active region as shown in Figure 3. In order to design more flexible gratings which are able to support a wider range of emission wavelengths, we decided to implement chirped grating DFBs (CGDFBs). Details are given in the Methods section. Such gratings are typically used in tunable DFB devices, due to their ability to simultaneously support a wide range of emission frequencies. ${ }^{30}$ Moreover, they are potentially beneficial for increasing the outcoupling efficiency compared to that of regular second order DFB structures. ${ }^{31}$

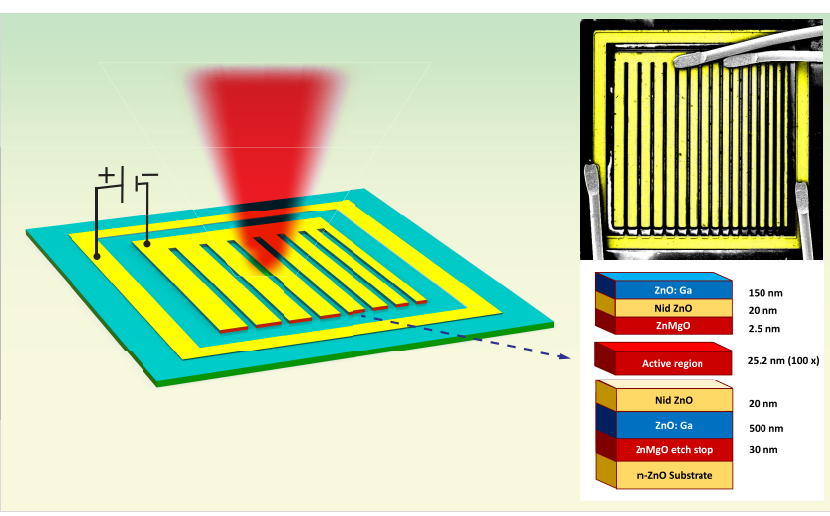

Figure 3. Schematic of the fabricated devices. The inset (top-right) shows the scanning electron microscope image of a typical device with bonded wires. The inset (bottom-right) shows the detailed waveguide structure.

Mesas with lateral sizes ranging from 100 to $400 \mu \mathrm{m}$ were implemented but we only present the analysis of the largest mesa in this work since it provides higher optical signal.

The electroluminescence spectra taken at a temperature $T=$ $110 \mathrm{~K}$ for different bias voltages are shown in Figure 4a. The
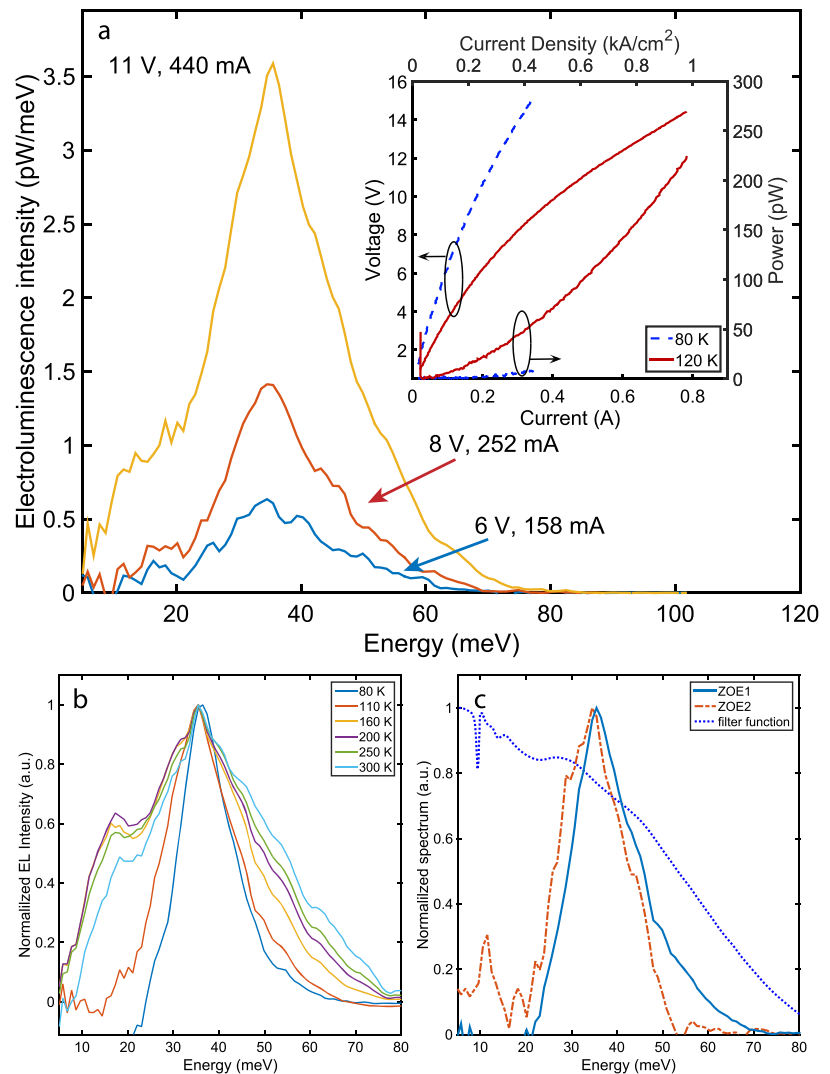

Figure 4. (a) Electroluminescence spectra of sample ZOE1 under different biases. The inset shows the power vs current $(L-I)$ and voltage vs current $(V-I)$ curves at temperatures of $80 \mathrm{~K}$ and $120 \mathrm{~K}$, respectively. (b) Temperature evolution of the electroluminescence spectra for temperatures from 80 to $300 \mathrm{~K}$. (c) Emission spectra of samples ZOE1 and ZOE2, and the filter transfer function of the bolometer. 
emission energy is $\sim 35 \mathrm{meV}$, with a full-width-at-maximum (fwhm) of $\sim 19 \mathrm{meV}$, which is comparable to the absorption line width observed in the $\mathrm{ZnO} / \mathrm{Zn}_{x} \mathrm{Mg}_{1-x} \mathrm{O}$ heterostructures. ${ }^{32}$ This is the first time that an electroluminescence peak has been observed at this frequency from an n-type $\mathrm{ZnO} /$ $\mathrm{Zn}_{x} \mathrm{Mg}_{1-x} \mathrm{O}$ quantum cascade structure. The observed emission energy is in reasonable agreement with the simulated center frequency of the gain spectrum $(24 \mathrm{meV})$ considering the uncertainties in the material parameters. It should be noted that similar discrepancies between the experimental and theoretical values have also been observed in the $\mathrm{THz}$ intersubband absorption of the nonpolar m-plane GaN-based systems. ${ }^{33,34}$ No Stark shifts or bias-dependent level broadening was detected in the studied voltage range. This was expected due to the large level broadening resulting from the strong LO-phonon scattering and interface roughness scattering in the $\mathrm{ZnO}$ QWs. The optical output power versus current $(L-I)$ and voltage versus current $(V-I)$ curves at temperatures of $80 \mathrm{~K}$ and $120 \mathrm{~K}$ are depicted in the inset of Figure $4 \mathrm{a}$. As reported for the SiGe material system, ${ }^{10}$ negative differential resistance (NDR) is not observed. This could be attributed the large level broadening which renders selective injection into the upper laser state less effective compared with the GaAs system. The electroluminescence (EL) spectrum has been measured at various temperatures with bias voltage fixed at $\sim 11 \mathrm{~V}$. The corresponding spectra taken from 80 to $300 \mathrm{~K}$ are shown in Figure 4b. Below $100 \mathrm{~K}$, a negative peaks appear in the emission spectra (see Supporting Information, Figure 1). The observed emission spectrum can be interpreted as the sum of two contributions: the intersubband emission and the modulated blackbody radiation from the hot bolometer window, with their relative weights determined by the operating temperature. The modulated blackbody radiation incident on the sample will be measured as a differential signal with a negative component. ${ }^{35}$ At low temperature, due to the small injected current, the modulated blackbody signal dominates over the intersubband emission, showing a strong negative peak in the emission spectrum. On the contrary, the intersubband emission starts to dominate with increasing temperature. The change in the relative weight of the two contributions can be recognized by the phase change of the emission spectrum (see Supporting Information, Figure 1). Therefore, no negative peaks are observed at high temperatures.

For comparison, the emission spectra for two samples, ZOE 1 and ZOE2 at a temperature of $100 \mathrm{~K}$ is shown in Figure $4 c$, together with the filter transfer function. The EL spectrum of ZOE2 with a slightly larger period shows a lower center frequency than that of ZOE1, which is consistent with the numerical simulation. Though the polarization of the emission is hard to determine with current grating structures, a distinguishable difference between the sample spectra and the filter transfer function, together with the reasonable agreement between the experiment and the theoretical energies demonstrate the intersubband nature of the sample emissions. We also noted that the gain disappears very fast with increasing interface roughness in our numerical simulation.

The line width of the spectrum increases from $12.7 \mathrm{meV}$ at $80 \mathrm{~K}$ to $36 \mathrm{meV}$ at $300 \mathrm{~K}$. The increased line width at high temperature is attributed to the stronger impurity scattering and electron-electron scattering as a result of the strong temperature dependence of the carrier density (see Supporting Information, Figure 2). In fact, the NEGF simulation at $300 \mathrm{~K}$ shows an energy level broadening almost twice as large as the broadening at $110 \mathrm{~K}$, which is consistent with the experimental results in Figure $4 \mathrm{~b}$. The detailed examination reveals that the line width at $80 \mathrm{~K}$ is limited at low energy (i.e., below $20 \mathrm{meV}$ ) by the modulated absorption signal described above, as indicated also by the asymmetry of the EL spectrum. Compared with GaAs $\mathrm{THz}$ QCLs, the sample shows the stronger temperature-dependence of the injection current, as depicted in the inset of Figure $4 a$. This can be explained by the large ionization energy of the $\mathrm{Ga}$ dopant in the $\mathrm{ZnO}$ heterostructure $^{36}$ (see Supporting Information, Figure 3). An ionization energy of $6.7 \mathrm{meV}$, corresponding to a temperature of $78 \mathrm{~K}$, was obtained for sample ZOE1, leading to a strong temperature-dependence of the free carrier density and, thus, the injected current. Given the above analysis, we exclude the electron-electron scattering as the main mechanism for the large broadening of the EL spectra at low temperature. ${ }^{37}$ Instead, we attribute it to LO phonon emission and interface roughness scattering. To make a quantitative comparison between the theory and experiment while suppressing thermal effect due to device heating, we performed the analysis at 110 K.

The EL power at $110 \mathrm{~K}$ can be written as

$$
P_{\text {opt }}=\eta_{\text {coll }} \eta_{\text {rad }} N_{\text {per }} \frac{I}{q_{0}} h v
$$

where $\eta_{\text {coll }}$ is collection efficiency of the experiment setup, $q_{0}$ is the electron charge, $N_{\text {per }}$ is the number of the active region periods, $h v$ is the photon energy, and $\eta_{\mathrm{rad}}=\tau_{\mathrm{nr}} / \tau_{\mathrm{rad}}$ is the quantum efficiency per period ( $\tau_{\mathrm{nr}}$ and $\tau_{\text {rad }}$ are the nonradiative and radiative lifetimes, respectively). ${ }^{10,37}$ Using $P_{\text {opt }} \sim 98 \mathrm{pW}$, $\eta_{\text {coll }} \sim 0.1 \%$, and $N_{\text {per }}=100$, we estimate a quantum efficiency of $\eta_{\text {rad }}=\sim 7.1 \times 10^{-8}$. With $\tau_{\text {rad }} \sim 20 \mu \mathrm{s}$, a nonradiative lifetime of $\tau_{\mathrm{nr}} \sim 1.3$ ps is estimated, which is in reasonable agreement with the calculated value by NEGF of $\tau_{\mathrm{nr}} \sim 2 \mathrm{ps}$. The quantum efficiency increases to $\sim 4.2 \times 10^{-7}$ at $300 \mathrm{~K}$ (Supporting Information, Figure 4), which can be attributed to both the stronger mean-field caused by higher carrier density and the blackbody radiation introduced by the hot electron plasmon in the structure. ${ }^{38}$ The estimated quantum efficiency is about 1 order of magnitude smaller than that obtained for the GaAs QCLs as a result of the short nonradiative lifetime. From the calculated upper laser state lifetime, we obtained a carrier density of $\sim 3 \times 10^{9} \mathrm{~cm}^{-2}$ for a current of $400 \mathrm{~mA}$ at $11 \mathrm{~V}$, which agrees well with the calculated value of $1 \times 10^{10} \mathrm{~cm}^{-2}$ (see Supporting Information, Figure 2). It should be emphasized that no fitting parameters were used in the simulations. However, reasonable agreement between the theory and the experiment in both the optical and electrical characteristics of the device has been achieved, which provides further evidence for the intersubband origin of the observed spectra. Given the much lower free carrier density than stateof-art GaAs $\mathrm{THz}$ QCLs ${ }^{8,39}$ and the strong Fröhlich interaction, ${ }^{40}$ we conclude that the electron-LO phonon and interface roughness are the main scattering mechanisms for the level broadening.

\section{CONCLUSIONS}

In conclusion, we have demonstrated $\mathrm{THz}$ electroluminescence from quantum cascade structures based on the nonpolar m-plane $\mathrm{ZnO} / \mathrm{Zn}_{88} \mathrm{Mg}_{12} \mathrm{O}$ material system. The electroluminescence shows a center frequency of $\sim 8.5 \mathrm{THz}$, which 
is unachievable in GaAs-based heterostructures owing to the intrinsic reststrahlen band absorption at $\sim 8 \mathrm{THz}$. The large ionization energy of the $\mathrm{Ga}$ dopant in $\mathrm{ZnO}$ leads to a strong temperature dependence of the free carrier density. Therefore, to observe a good signal-to-noise ratio, the device has to be operated above the ionization temperature. In this respect, somewhat counterintuitively, the device shows enhanced emission intensity with increasing temperature. At low temperature, the line width broadening is attributed to the electron-LO phonon and interface roughness scatterings due to the low free carrier density. However, as the temperature increases, the electroluminescence spectra become significantly broadened due to stronger impurity and electron-electron scatterings, as both scattering mechanisms show strong dependence on the carrier density. To further improve the performance of the device, or even achieve lasing action, first, the strong temperature dependence of the injection current has to be mitigated. Potential methods include using higher doping levels or modulation doping in the barriers. Second, as the strong electron-LO phonon scattering strongly broadens the energy levels, an extraction well with extraction energy that is not resonant with the LO phonon will reduce the level broadening while keeping a fast extraction rate from the lower laser state. Meanwhile, structures with lower transition energies could benefit due to the reduced free carrier and intrinsic material absorptions. In addition, our simulations show that reducing the interface roughness could significantly enhance the gain of the active region. To summarize, our demonstration not only constitutes a significant step further toward the realization of $\mathrm{ZnO}$-based $\mathrm{THz}$ QCLs, but also unlocks the potential of $\mathrm{ZnO}$-based heterostructures for novel devices and fundamental physics investigations, such as mid-infrared lasers and phonon-polariton emitters. ${ }^{41}$

\section{METHODS}

Active Region and Grating Designs. For the calculations, the band offset between the $\mathrm{QW}$ and the barrier was taken as $\Delta E_{\mathrm{c}}=0.675 \Delta E_{\mathrm{g}}$, where $\Delta E_{\mathrm{g}}$ is the bandgap difference between $\mathrm{ZnO}$ and $\mathrm{Zn}_{1-x} \mathrm{Mg}_{x} \mathrm{O}$ and was calculated as $25 \mathrm{meV}$ per $\%$ of $\mathrm{Mg}$ in the barrier. ${ }^{21}$ The effective masses of the QWs and the barriers were taken to be the same as that of $\mathrm{ZnO}$ $\left(0.22 m_{0}\right)$, where $m_{0}$ is the free electron mass. This assumption does not introduce a significant error since the $\mathrm{Mg}$ content has been fixed at $12 \%$ to lead to a conduction band offset of $\sim 200$ $\mathrm{meV}$.

Our gratings are designed with a linearly chirped periodicity, ranging from $15.2 \mu \mathrm{m}$ up to $31.2 \mu \mathrm{m}$ (average of $24.7 \mu \mathrm{m}$ ). In contrast, the grooves of the grating have an identical length of $6.2 \mu \mathrm{m}$ per period. We further enhanced the outcoupling strength of the gratings by etching them through the active region (down to the bottom $500 \mathrm{~nm} \mathrm{ZnO}$ :Ga waveguide $(3 \times$ $\left.10^{18} \mathrm{~cm}^{-3}\right)$. To allow for efficient light outcoupling, only the top of the gratings were covered with gold for current injection. Based on the general numerical method for grating efficiency coupling, ${ }^{31}$ the proposed CGDFBs were estimated to support light extraction for wavelengths ranging from 2.4 to 19.7 THz.

Growth Procedure. We grew the sample on a $10 \times 20$ $\mathrm{mm}^{2} \mathrm{ZnO}$ m-plane substrate. We annealed it prior to growth with a rapid thermal annealing system at $1065{ }^{\circ} \mathrm{C}$ for $2 \mathrm{~min}$ under an oxygen atmosphere. This procedure is known to reveal atomic steps on the surface. ${ }^{42}$ For the growth, we used a molecular beam epitaxy system Compact 21 from Riber equipped with $2 \mathrm{Zn}, 2 \mathrm{Mg}$, and $2 \mathrm{Ga}$ effusion cells and $2 \mathrm{O}$ plasma cells. We adjusted the $\mathrm{Zn}$ and $\mathrm{O}$ fluxes to be close to the stoichiometry to limit point defects ${ }^{43}$ and set the sample temperature to $165^{\circ} \mathrm{C}$. Usually, the $\mathrm{MBE}$ growth of $\mathrm{ZnO}$ on nonpolar orientations like m-plane was performed at low temperature in order to avoid the phase transition of $\mathrm{ZnMgO}$ (from wurtzite to rocksalt). Moreover, the $\mathrm{Zn}$ sticking coefficient is low and decreases very quickly for temperatures above $400{ }^{\circ} \mathrm{C}$. With these growth conditions, the growth rate is $170 \mathrm{~nm} / \mathrm{h}$. Growing cascade structures for the THz domain is very material demanding. Indeed the thickness control needs to be under the monolayer $(\mathrm{ML})$ scale $(0.28 \mathrm{~nm}$ for m-plane $\mathrm{ZnO}$ ). Otherwise a huge energy shift appears between the measured and the targeted intersubband transition energies. We set a fine calibration procedure to tackle this very strict requirement. ${ }^{22}$ It involves a specifically designed calibration sample and a combination of the scanning electron microscopy and X-ray reflectivity experiments for thickness determination. By doing so, we achieved a thickness determination with a sub$\mathrm{ML}$ precision even for thicknesses down to $2 \mathrm{ML}$. We crosschecked these results by a high resolution scanning transmission electron microscopy (HRSTEM) experiment. It is shown that most of the layers within the active region period are close to being an integer number of monolayer, except for the $2.4 \mathrm{~nm}$ barrier layer, with a roughness of $0.168 \mathrm{~nm}$. This is the only layer in the active region showing a roughness higher than the $0.1 \mathrm{~nm}$, estimated from the XRR. In fact, the interface roughness is estimated to be clearly less than one monolayer, as obtained from the HRSTEM images.

Device Fabrication. The whole device fabrication scheme consists of multiple steps, including the etching of the implemented CGDFBs into the m-plane $\mathrm{ZnO} / \mathrm{ZnMgO}$ layer structure in a $\mathrm{CH}_{4} / \mathrm{H}_{2} / \mathrm{Ar}$ RIE-plasma, followed by a wet (smoothing) etch in diluted $\mathrm{HCl}$ (details given elsewhere ${ }^{44}$ ). In order to significantly reduce surface leakage effects, wellknown to etched $\mathrm{ZnO}$-based structures, ${ }^{45}$ the sample was treated with $\mathrm{H}_{2} \mathrm{O}_{2}$ for $5 \mathrm{~min}$ at $95{ }^{\circ} \mathrm{C}$. This forms an oxygenrich top-layer ${ }^{46}$ and cures deep level defect states ${ }^{47}$ in the etched $\mathrm{ZnO}$ surface, strongly reducing the carrier concentration at the sample surface. Hence, leakage currents are effectively suppressed by more than 2 orders of magnitude. ${ }^{44}$ The hydrogen peroxide passivation is followed by a covering the entire sample with $1.6 \mu \mathrm{m}$ of $\mathrm{SiN}$ to protect and passivate the sample surface, only exposing the electrical contact area. In the last step $\mathrm{Ti} / \mathrm{Au}$ (thickness: $10 \mathrm{~nm} / 200 \mathrm{~nm}$ ) contacts are sputtered on top of the sample. The right-top inset of Figure 3 shows a scanning electron microscope image of a typical final device.

Optical Measurement. The cleaved devices were indium soldered on copper submounts, wire bonded, and mounted on the coldfinger of a He flow cryostat. Current pulses with pulse widths between $200 \mathrm{~ns}$ and $1.7 \mu \mathrm{s}$ and a constant interval of $1.91 \mu$ s were applied. In addition, a modulation at $415 \mathrm{~Hz}$ was used to match the frequency response of the $\mathrm{Si}$ bolometer while minimizing the thermal effect from device heating. The emission from the devices was collected by an $f / 1$ off-axis parabolic mirror and sent into a home-built vacuum Fourier transform infrared spectrometer (FTIR) equipped with a Sibolometer for spectrum and intensity measurements. The emitted light was collected in the step scan mode using the lock-in technique. The phase of the lock-in amplifier was prior calibrated by a surface emission $\mathrm{THz}$ QCL. ${ }^{48}$ 


\section{ASSOCIATED CONTENT}

\section{(s) Supporting Information}

The Supporting Information is available free of charge at https://pubs.acs.org/doi/10.1021/acsphotonics.0c01641.

Details on the emission spectrum at low temperature, temperature dependence of free carrier concentration, ionization energy of $\mathrm{Ga}$, and temperature dependence of emission power and quantum efficiency (PDF)

\section{AUTHOR INFORMATION}

\section{Corresponding Authors}

Bo Meng - Institute for Quantum Electronics, ETH Zurich, Zurich 8093, Switzerland; $\odot$ orcid.org/0000-0002-96490846; Email: bmeng@phys.ethz.ch

Borislav Hinkov - Institute of Solid State Electronics and Center for Micro and Nanostructures, TU Wien, 1040 Vienna, Austria; Email: borislav.hinkov@tuwien.ac.at

Jérôme Faist - Institute for Quantum Electronics, ETH Zurich, Zurich 8093, Switzerland; Email: jerome.faist@ phys.ethz.ch

Jean M. Chauveau - Université Côte d'Azur, CNRS, CRHEA, 06560 Valbonne, France; Email: jean.michel.chauveau@ crhea.cnrs.fr

\section{Authors}

Nolwenn Marie L. Biavan - Université Côte d'Azur, CNRS, CRHEA, 06560 Valbonne, France

Hanh T. Hoang - Institute of Solid State Electronics and Center for Micro and Nanostructures, TU Wien, 1040 Vienna, Austria

Denis Lefebvre - Université Côte d'Azur, CNRS, CRHEA, 06560 Valbonne, France

Maxime Hugues - Université Côte d'Azur, CNRS, CRHEA, 06560 Valbonne, France

David Stark - Institute for Quantum Electronics, ETH Zurich, Zurich 8093, Switzerland

Martin Franckié - Institute for Quantum Electronics, ETH Zurich, Zurich 8093, Switzerland

Almudena Torres-Pardo - Departamento de Química Inorgánica, Universidad Complutense de Madrid, 28040 Madrid, Spain; ๑ orcid.org/0000-0002-1667-4072

Julen Tamayo-Arriola - ISOM, Universidad Politécnica de Madrid, 28040 Madrid, Spain

Miguel M. Bajo - ISOM, Universidad Politécnica de Madrid, 28040 Madrid, Spain; ๑ orcid.org/0000-0002-9352-5820

Adrian Hierro - ISOM, Universidad Politécnica de Madrid, 28040 Madrid, Spain

Gottfried Strasser - Institute of Solid State Electronics and Center for Micro and Nanostructures, TU Wien, 1040 Vienna, Austria

Complete contact information is available at:

https://pubs.acs.org/10.1021/acsphotonics.0c01641

\section{Notes}

The authors declare no competing financial interest.

\section{ACKNOWLEDGMENTS}

This project has been funded by the European Union's Horizon 2020 Research and Innovation Program under Grant Agreement No. 665107 (ZOTERAC); European Union's Horizon 2020 Research and Innovation Program under Grant Agreement No. 766719 (FLASH); European Union's
Horizon 2020 Research and Innovation Program under Grant Agreement No. 820419 (Qombs).

\section{REFERENCES}

(1) Williams, B. S. Terahertz quantum-cascade lasers. Nat. Photonics 2007, 1, 517-525.

(2) Scalari, G.; Walther, C.; Fischer, M.; Terazzi, R.; Beere, H.; Ritchie, D.; Faist, J. THz and sub-THz quantum cascade lasers. Laser Photonics Rev. 2009, 3, 45-66.

(3) Köhler, R.; Tredicucci, A.; Beltram, F.; Beere, H. E.; Linfield, E. H.; Davies, A. G.; Ritchie, D. A.; Iotti, R. C.; Rossi, F. Terahertz semiconductor-heterostructure laser. Nature 2002, 417, 156-159.

(4) Maestrini, A.; Thomas, B.; Wang, H.; Jung, C.; Treuttel, J.; Jin, Y.; Chattopadhyay, G.; Mehdi, I.; Beaudin, G. Schottky diode-based terahertz frequency multipliers and mixers. C. R. Phys. 2010, 11, 480495.

(5) Khalid, A.; Dunn, G. M.; Macpherson, R. F.; Thoms, S.; Macintyre, D.; Li, C.; Steer, M. J.; Papageorgiou, V.; Thayne, I. G.; Kuball, M.; Oxley, C. H.; Bajo, M. M.; Stephen, A.; Glover, J.; Cumming, D. R. S. Terahertz oscillations in an $\mathrm{In} 0_{.53} \mathrm{Ga}_{0.47}$ As submicron planar Gunn diode. J. Appl. Phys. 2014, 115, 114502.

(6) Kasagi, K.; Suzuki, S.; Asada, M. Large-scale array of resonanttunneling-diode terahertz oscillators for high output power at $1 \mathrm{THz}$. J. Appl. Phys. 2019, 125, 151601.

(7) Berry, C. W.; Wang, N.; Hashemi, M. R.; Unlu, M.; Jarrahi, M. Significant performance enhancement in photoconductive terahertz optoelectronics by incorporating plasmonic contact electrodes. Nat. Commun. 2013, 4, 1622.

(8) Bosco, L.; Franckié, M.; Scalari, G.; Beck, M.; Wacker, A.; Faist, $\mathrm{J}$. Thermoelectrically cooled $\mathrm{THz}$ quantum cascade laser operating up to 210 K. Appl. Phys. Lett. 2019, 115, 010601.

(9) Franckié, M.; Faist, J. Bayesian Optimization of Terahertz Quantum Cascade Lasers. Phys. Rev. Appl. 2020, 13, 034025.

(10) Dehlinger, G.; Diehl, L.; Gennser, U.; Sigg, H.; Faist, J.; Ensslin, K.; Grützmacher, D.; Müller, E. Intersubband Electroluminescence from Silicon-Based Quantum Cascade Structures. Science 2000, 290, $2277-2280$

(11) Terashima, W.; Hirayama, H. Spontaneous emission from $\mathrm{GaN} / \mathrm{AlGaN}$ terahertz quantum cascade laser grown on $\mathrm{GaN}$ substrate. Phys. Status Solidi c 2011, 8, 2302-2304.

(12) Bellotti, E.; Paiella, R. Numerical Simulation of ZnO-Based Terahertz Quantum Cascade Lasers. J. Electron. Mater. 2010, 39, 1097-1103.

(13) Sirkeli, V. P.; Hartnagel, H. L. ZnO-based terahertz quantum cascade lasers. Opto-Electron. Rev. 2019, 27, 119-122.

(14) Chou, H. C.; Mazady, A.; Zeller, J.; Manzur, T.; Anwar, M. Room-Temperature Quantum Cascade Laser: $\mathrm{ZnO} / \mathrm{Zn}_{1-\mathrm{x}} \mathrm{Mg}_{\mathrm{x}} \mathrm{O}$ Versus GaN/ $\mathrm{Al}_{\mathrm{x}} \mathrm{Ga}_{1-\mathrm{x}} \mathrm{N}$. J. Electron. Mater. 2013, 42, 882-888.

(15) Grange, T.; Stark, D.; Scalari, G.; Faist, J.; Persichetti, L.; Di Gaspare, L.; De Seta, M.; Ortolani, M.; Paul, D. J.; Capellini, G.; Birner, S.; Virgilio, M. Room temperature operation of n-type Ge/ $\mathrm{SiGe}$ terahertz quantum cascade lasers predicted by non-equilibrium Green's functions. Appl. Phys. Lett. 2019, 114, 111102.

(16) Albo, A.; Flores, Y. V.; Hu, Q.; Reno, J. L. Split-well directphonon terahertz quantum cascade lasers. Appl. Phys. Lett. 2019, 114, 191102.

(17) Tsukazaki, A.; Ohtomo, A.; Onuma, T.; Ohtani, M.; Makino, T.; Sumiya, M.; Ohtani, K.; Chichibu, S. F.; Fuke, S.; Segawa, Y.; Ohno, H.; Koinuma, H.; Kawasaki, M. Repeated temperature modulation epitaxy for p-type doping and light-emitting diode based on ZnO. Nat. Mater. 2004, 4, 42-46.

(18) Tsukazaki, A.; Akasaka, S.; Nakahara, K.; Ohno, Y.; Ohno, H.; Maryenko, D.; Ohtomo, A.; Kawasaki, M. Observation of the fractional quantum Hall effect in an oxide. Nat. Mater. 2010, 9, 889-893.

(19) Frenzel, H.; Lajn, A.; von Wenckstern, H.; Lorenz, M.; Schein, F.; Zhang, Z.; Grundmann, M. Recent Progress on ZnO-Based MetalSemiconductor Field-Effect Transistors and Their Application in Transparent Integrated Circuits. Adv. Mater. 2010, 22, 5332-5349. 
(20) Le Biavan, N.; Hugues, M.; Bajo, M. M.; Tamayo-Arriola, J.; Jollivet, A.; Lefebvre, D.; Cordier, Y.; Vinter, B.; Julien, F.-H.; Hierro, A.; Chauveau, J.-M. Homoepitaxy of non-polar $\mathrm{ZnO} /(\mathrm{Zn}, \mathrm{Mg}) \mathrm{O}$ multi-quantum wells: From a precise growth control to the observation of intersubband transitions. Appl. Phys. Lett. 2017, 111, 231903.

(21) Montes Bajo, M.; Tamayo-Arriola, J.; Hugues, M.; Ulloa, J. M.; Le Biavan, N.; Peretti, R.; Julien, F. H.; Faist, J.; Chauveau, J.-M.; Hierro, A. Multisubband Plasmons in Doped ZnO Quantum Wells. Phys. Rev. Appl. 2018, 10, 024005.

(22) Meng, B.; Tamayo-Arriola, J.; Le Biavan, N.; Montes Bajo, M.; Torres-Pardo, A.; Hugues, M.; Lefebvre, D.; Hierro, A.; Chauveau, J.M.; Faist, J. Observation of Intersubband Absorption in $\mathrm{ZnO}$ Coupled Quantum Wells. Phys. Rev. Appl. 2019, 12, 054007.

(23) Wacker, A.; Lindskog, M.; Winge, D. O. Nonequilibrium Green's Function Model for Simulation of Quantum Cascade Laser Devices Under Operating Conditions. IEEE J. Sel. Top. Quantum Electron. 2013, 19, 1-11.

(24) Ohtani, K.; Turčinková, D.; Bonzon, C.; Benea-Chelmus, I.-C.; Beck, M.; Faist, J.; Justen, M.; Graf, U. U.; Mertens, M.; Stutzki, J. High performance $4.7 \mathrm{THz}$ GaAs quantum cascade lasers based on four quantum wells. New J. Phys. 2016, 18, 123004.

(25) Amanti, M. I.; Scalari, G.; Terazzi, R.; Fischer, M.; Beck, M.; Faist, J.; Rudra, A.; Gallo, P.; Kapon, E. Bound-to-continuum terahertz quantum cascade laser with a single-quantum-well phonon extraction/injection stage. New J. Phys. 2009, 11, 125022.

(26) Grange, T.; Stark, D.; Scalari, G.; Faist, J.; Persichetti, L.; Di Gaspare, L.; De Seta, M.; Ortolani, M.; Paul, D. J.; Capellini, G.; Birner, S.; Virgilio, M. Room temperature operation of n-type Ge/ $\mathrm{SiGe}$ terahertz quantum cascade lasers predicted by non-equilibrium Green's functions. Appl. Phys. Lett. 2019, 114, 111102.

(27) Moram, M. A.; Vickers, M. E. X-ray diffraction of III-nitrides. Rep. Prog. Phys. 2009, 72, 036502.

(28) Faist, J.; Capasso, F.; Sivco, D. L.; Sirtori, C.; Hutchinson, A. L.; Cho, A. Y. Quantum Cascade Laser. Science 1994, 264, 553-556.

(29) Xu, B.; Hu, Q.; Melloch, M. R. Electrically pumped tunable terahertz emitter based on intersubband transition. Appl. Phys. Lett. 1997, 71, 440-442.

(30) Chen, N.; Nakano, Y.; Okamoto, K.; Tada, K.; Morthier, G. I.; Baets, R. G. Analysis, fabrication, and characterization of tunable DFB lasers with chirped gratings. IEEE J. Sel. Top. Quantum Electron. 1997, $3,541-546$.

(31) Xu, B.; Hu, Q. Grating coupling for intersubband emission. Appl. Phys. Lett. 1997, 70, 2511-2513.

(32) Jollivet, A. Private communication.

(33) Edmunds, C.; Shao, J.; Shirazi-HD, M.; Manfra, M. J.; Malis, O. Terahertz intersubband absorption in non-polar m-plane AlGaN/ GaN quantum wells. Appl. Phys. Lett. 2014, 105, 021109.

(34) Lim, C. B.; Ajay, A.; Bougerol, C.; Haas, B.; Schörmann, J.; Beeler, M.; Lähnemann, J.; Eickhoff, M.; Monroy, E. Nonpolarmplane $\mathrm{GaN} / \mathrm{AlGaN}$ heterostructures with intersubband transitions in the 5-10 THz band. Nanotechnology 2015, 26, 435201.

(35) Geiser, M.; Beck, M.; Faist, J. Terahertz intersubband polariton tuning by electrical gating. Opt. Express 2014, 22, 2126-2131.

(36) Brochen, S.; Feuillet, G.; Santailler, J.-L.; Obrecht, R.; Lafossas, M.; Ferret, P.; Chauveau, J.-M.; Pernot, J. Non-metal to metal transition in n-type $\mathrm{ZnO}$ single crystal materials. J. Appl. Phys. 2017, 121, 095704.

(37) Rochat, M.; Faist, J.; Beck, M.; Oesterle, U.; Ilegems, M. Farinfrared $(\lambda=88 \mu \mathrm{m})$ electroluminescence in a quantum cascade structure. Appl. Phys. Lett. 1998, 73, 3724-3726.

(38) Hirakawa, K.; Grayson, M.; Tsui, D. C.; Kurdak, Ç. Blackbody radiation from hot two-dimensional electrons in $\mathrm{Al}_{\mathrm{x}} \mathrm{Ga}_{1-\mathrm{x}} \mathrm{As} / \mathrm{GaAs}$ heterojunctions. Phys. Rev. B: Condens. Matter Mater. Phys. 1993, 47, 16651-16654.

(39) Fathololoumi, S.; Dupont, E.; Chan, C. W. I.; Wasilewski, Z. R.; Laframboise, S. R.; Ban, D.; Mátyás, A.; Jirauschek, C.; Hu, Q.; Liu, H. C. Terahertz quantum cascade lasers operating up to $\sim 200 \mathrm{~K}$ with optimized oscillator strength and improved injection tunneling. Opt. Express 2012, 20, 3866-3876.

(40) Yasuda, H.; Kubis, T.; Hosako, I.; Hirakawa, K. Nonequilibrium Green's function calculation for GaN-based terahertzquantum cascade laser structures. J. Appl. Phys. 2012, 111, 083105.

(41) Ohtani, K.; Meng, B.; Franckié, M.; Bosco, L.; NdebekaBandou, C.; Beck, M.; Faist, J. An electrically pumped phononpolariton laser. Sci. Adv. 2019, 5, eaau1632.

(42) Ko, H.-J.; Han, M.-S.; Park, Y.-S.; Yu, Y.-S.; Kim, B.-I.; Kim, S. S.; Kim, J.-H. Improvement of the quality of $\mathrm{ZnO}$ substrates by annealing. J. Cryst. Growth 2004, 269, 493-498.

(43) Arthur, J. R. Molecular beam epitaxy. Surf. Sci. 2002, 500, 189217.

(44) Hinkov, B. Manuscript in preparation.

(45) Lee, J.-M.; Kim, K.-K.; Park, S.-J.; Choi, W.-K. Low-resistance and nonalloyed ohmic contacts to plasma treated $\mathrm{ZnO}$. Appl. Phys. Lett. 2001, 78, 3842-3844.

(46) Schifano, R.; Monakhov, E. V.; Svensson, B. G.; Diplas, S. Surface passivation and interface reactions induced by hydrogen peroxide treatment of n-type $\mathrm{ZnO}(000 \overline{1})$. Appl. Phys. Lett. 2009, 94, 132101.

(47) Kim, S.-H.; Kim, H.-K.; Seong, T.-Y. Effect of hydrogen peroxide treatment on the characteristics of Pt Schottky contact on ntype ZnO. Appl. Phys. Lett. 2005, 86, 112101.

(48) Bosco, L.; Bonzon, C.; Ohtani, K.; Justen, M.; Beck, M.; Faist, J. A patch-array antenna single-mode low electrical dissipation continuous wave terahertz quantum cascade laser. Appl. Phys. Lett. 2016, 109, 201103. 